\title{
The Hot Components in the Recurrent Novae $T$ Pyxidis, IM Norma, Cl Aquilae and in the Dwarf Nova U Geminorum
}

\author{
Edward M. Sion* and Patrick Godon ${ }^{\dagger}$ \\ Astrophysics \& Planetary Science \\ Villanova University \\ Villanova, PA 19085, USA \\ E-mail: edward.sion@villanova.edu, patrick.godon@villanova.edu
}

\begin{abstract}
We report on the latest results from Hubble Space Telescope (HST) far ultraviolet spectroscopy with the Cosmic Object Spectrograph (COS), of the short orbital period recurrent novae (RNe) T Pyxidis, IM Norma (the first ever FUV spectrum of this RN) and CI Aquilae, the only known recurrent novae with main sequence donors and orbital periods less than a day. The last two HST COS spectra we obtained in October, 2016 and June, 2016, reveal that the accretion rate of T Pyx has declined by $20 \%$ below its quiescent continuum flux level recorded by the IUE following the 1966 outburst, an indication that the system might not have yet reached its (new) quiescent state. Recent HST COS Spectroscopy of the cooling of the white dwarf in the prototypical dwarf nova $\mathrm{U}$ Geminorum is also discussed with a focus on the chemical abundances in the white dwarf photosphere.
\end{abstract}

The Golden Age of Cataclysmic Variables and Related Objects IV

11-16 September, 2017

Palermo, Italy

\footnotetext{
* Speaker.

$\dagger$ Visiting in the Henry A. Rowland Department of Physics \& Astronomy, Johns Hopkins University, Baltimore, MD 21218, USA
} 


\section{The Short Orbital Period T Pyx Subclass of Recurrent Novae}

The recurrent novae ( $\mathrm{RNe}$ ) are a subset of $\mathrm{CVs}$ that have suffered more than one recorded outburst on recurrence timescales of a year to a century. Recent reviews on the properties and basic parameters of RNe can be found in $[1,2,16]$. Their short recurrence time between outbursts require both a massive WD and a high accretion rate [22, 9, 25]. These two factors are the critical ingredients needed for an accreting WD to reach the Chandrasekhar mass limit and explode as a Type Ia supernova (SN Ia), which is why RNe are among the best possibilities for a solution to the longstanding SN Ia progenitor problem [13].

However, a small subclass of RNe (T Pyx, IM Nor, CI Aql) have short orbital periods and main sequence (MS) donors stars like CVs. All other RNe have evolved (subgiant or red giant) donor companions and long orbital periods (days to years). In the following three subsections we describe the latest HST far Ultraviolet spectroscopy of T Pyx and optical and X-ray studies of IM Nor and CI Aql. Both IM Nor and CI Aql have had no previous FUV spectroscopy. In Table 1, we provide the physical and orbital parameters of the three systems from the literature, in some cases with large uncertainties.

Table 1: The T Pyx Subclass of Recurrent Novae

\begin{tabular}{cccccl}
\hline $\begin{array}{c}\text { System } \\
\text { Name }\end{array}$ & $\begin{array}{c}\mathrm{V} \\
\max \end{array}$ & $\begin{array}{c}\mathrm{V} \\
\min \end{array}$ & $\begin{array}{c}\mathrm{t} 3 \\
(\mathrm{~d})\end{array}$ & $\begin{array}{c}P_{\text {orb }} \\
(\mathrm{d})\end{array}$ & $\begin{array}{l}\text { Outbursts } \\
(\text { year })\end{array}$ \\
\hline T Pyx & 6.4 & 15.5 & 62 & 0.076 & $1890,1902,1920,1944,1966,2011$ \\
IM Nor & 8.5 & 18.3 & 80 & 0.102 & 1920,2002 \\
CI Aql & 9.0 & 16.7 & 32 & 0.62 & $1917,1941,2000$ \\
\hline
\end{tabular}

\subsection{T Pyxidis (Nova Pyxidis 1890)}

The first FUV spectra taken with IUE were 14 years after the 1966 outburst. T Pyx had already reached deep quiescence after its 2011 outburst when the flux level of the 2013 HST observations reached the continuum flux level of the IUE spectra taken in the 1980's and 1990's. To our surprise, our Oct 2015 and June 2016 COS spectra of T Pyx (Cycle 23) revealed a further 20\% drop in the FUV flux (Fig.1), with the June 2016 flux being slightly above the Oct 2016 but still well below the IUE quiescence flux level. Remarkably, a strong O I + Si III (1300) emission line and strong broad N V $(1238,1242)$ absorption lines appeared for the first time

From our model continuum slope fitting, and the light echo distance of $4.8 \mathrm{kpc}$, [4] we found that the quiescent mass accretion rate in T Pyx is very high (of the order of $10^{-6} M_{\odot} / \mathrm{yr}$ ). However, the accretion rate depends strongly on the distance: the very high accretion rate of $10^{-6} M_{\odot} / \mathrm{yr}$ assumes the light echo distance of $4.8 \mathrm{kpc}$ [21], while in preliminary study assuming a distance of only $\sim 1 \mathrm{kpc}$ we obtained $\dot{M}=10^{-8} M_{\odot} / \mathrm{yr}$ [17]. Such a large mass accretion rate implies that the inner disk/boundary layer/white dwarf is very hot and hence must be a source of strong soft $\mathrm{X}$-rays and EUV emission. However, this expected very hot soft-Xray/EUV source is not detected. The question then becomes how such a source of emission could be hidden. Two possibilities have emerged; (1) The hot source either emits intense soft X-ray/EUV radiation which is at so great a 


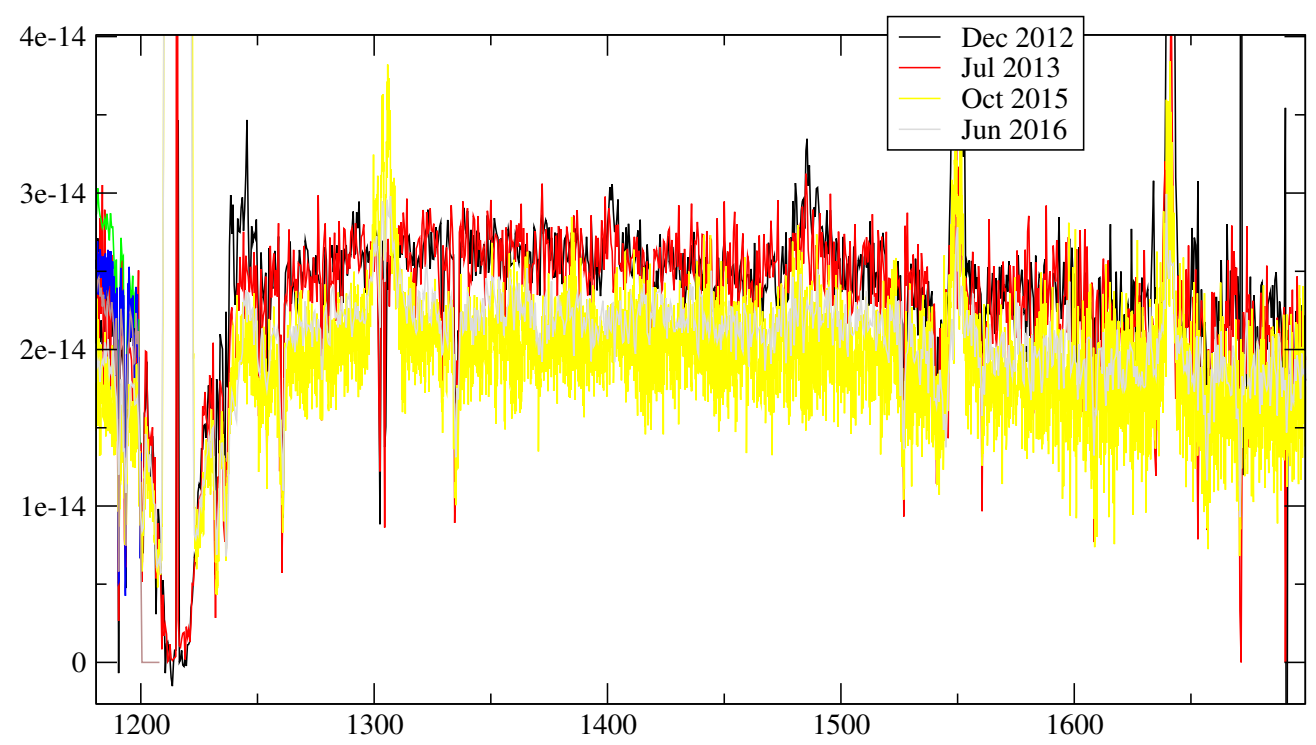

Figure 1: The last four HST FUV spectra of T Pyx have been displayed in color for clarity. The 2012 \& 2013 spectra have the same continuum flux level as the (1980-1996) IUE SWP spectra (not shown here), which was interpreted as having at last reached quiescence. However, the 2015 spectrum showed a further drop in the flux level by about $20 \%$, while the 2016 spectrum seems to show a slight increase in flux level. Between 1980 and 1996 the IUE spectra of T Pyx showed an average variation of only about 5\%, indicating that the present change is more than a fluctuation.

distance $(4.8 \mathrm{kpc})$, that it is completely absorbed by the interstellar medium, or (2) the hot inner disk/BL/WD soft X-ray/EUV emission is hidden by the upward flaring of the accretion disk outer edges when viewed at a high system inclination.

At this time, we are unable to state unequivocally whether the white dwarf mass is increasing or decreasing with time. This issue must await the availability of a GAIA distance, an upcoming Hubble Cycle 25 COS observation of T Pyx, and further analysis based upon the assumed reddening $\mathrm{E}(\mathrm{B}-\mathrm{V})$ of the system, GAIA parallax, and non-standard as well as standard disk models.

\subsection{IM Norma (Nova Nor 1920)}

The 2002 outburst of the RN IM Nor was followed visually and spectroscopically. In the beginning of the outburst, the nova belonged to Williams' Fe II class, but one month later, the spectrum changed to the $\mathrm{He} / \mathrm{N}$ class. It was detected as a hard $\mathrm{X}$-ray source 6 months after the 2002 outburst [12]. [24] found its $P_{\text {orb }}$ to be $2.46 \mathrm{~h}$ which places IM Nor in the middle of the CV period gap, an interval in the distribution of $\mathrm{CV}$ orbital periods between $2 \mathrm{hr}$ and $3 \mathrm{hr}$ where very few CVs are found.

The distance to IM Nor was determined using the Maximum Magnitude-Rate of Decline (MMRD) relation [16]. Its light curve remained fairly constant into quiescence. One possible 
explanation for this near constancy is that, like T Pyx, IM Nor has a persistent supersoft X-ray source which heats its companion star so as to drive the high mass accretion rate [10,16]. That is, IM Nor has a short orbital period, but ordinary angular momentum loss mechanisms cannot drive the accretion at the high rate required to power the short recurrence time scale.

The first ever FUV spectrum of IM Nor (an HST COS spectrum) is displayed in Fig.2. No absorption features are clearly detectable, in part due to the low $\mathrm{S} / \mathrm{N}$ of the spectrum but several emission features are seen, the strongest of which are Ly $\alpha$ and O I + Si III (1300) along with some relatively weak emission features including $\mathrm{NV}(1238,1242)$, both components of the resonance doublet C IV $(1548,1550)$ and the recombination line at He II (1640). The analysis of this spectrum awaits a GAIA parallax distance and will be reported elsewhere.

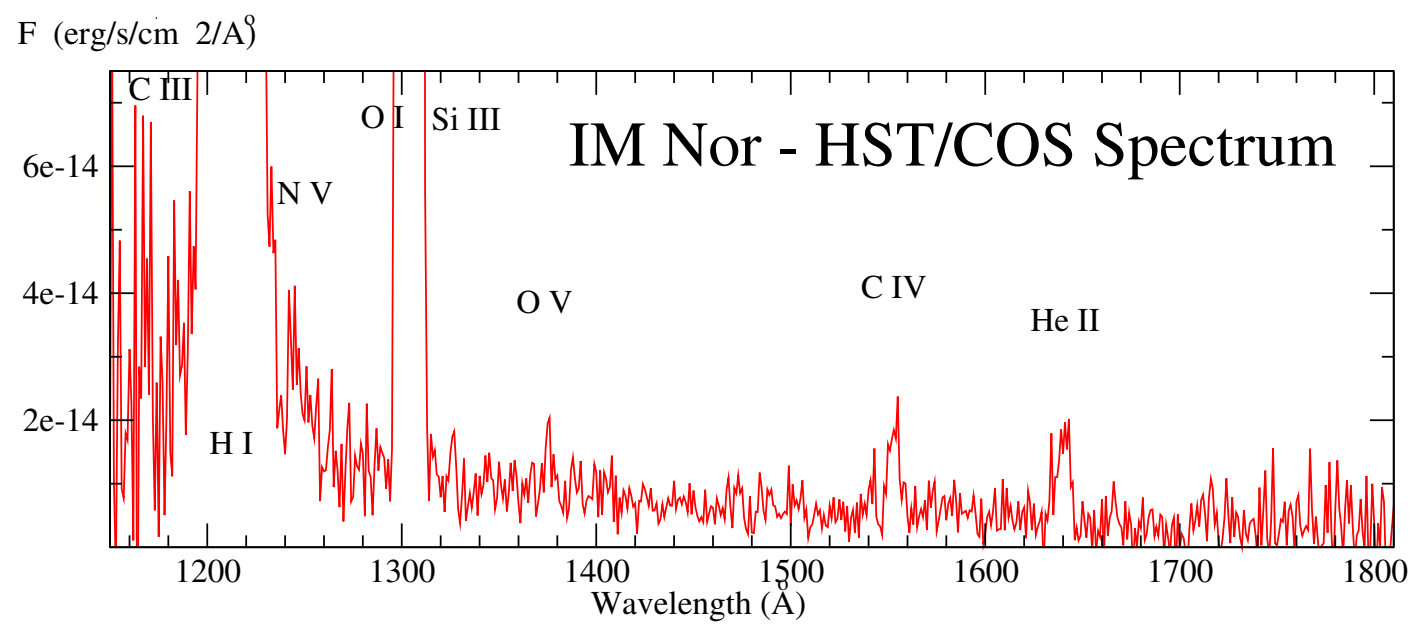

Figure 2: An HST COS spectrum of IM Nor (Flux versus wavelength) showing strong Ly $\alpha$ emission, strong O I + Si III (1300) and weak N V $(1238,1242)$, both components of the resonance doublet C IV $(1548,1550)$ and He II (1640). Note that the FUV continuum rises toward shorter wavelengths as expected for a hot source.

\subsection{Aquilae (Nova Aql 1917)}

[8] showed that its spectral evolution during the outburst resembled that of T Pyx type RNe. [11] found that CI Aql shows eclipses on an orbital period of $14.8 \mathrm{~h}$, with a K-M evolved or MS donor star. Its optical quiescence spectrum is very different from classical nova systems. The spectrum shows weak emission lines due to He II, and the C III - N III complex on a reddened spectrum [2]. [8] finds that its distance does not exceed $1 \mathrm{kpc}$ which implies that its donor is a MS star.

CI Aql was detected as a soft X-ray source, 14 and 16 months after the 2000 outburst [7]. From radial velocities, [14] find the mass of the WD to be $1.00 \pm 0.14 M_{\odot}$ and the mass of the donor secondary star to be $2.32 \pm 0.19 M_{\odot}$. They estimate the radius of the secondary to be $2.07 \pm 0.06 R_{\odot}$, implying that it is a slightly evolved early A-type star. The high mass ratio of $q=2.35 \pm 0.24$ and 
the high secondary-star mass implies that the mass transfer occurs on a thermal time-scale. [15] suggest that CI Aql may be evolving into a persistent supersoft x-ray source, and will eventually explode as an SN Ia. Models also suggest it may ultimately explode as a SNIa, possibly within 10 Myr [14]. Moreover, [23] found that the rate of period change, $d P / d t$, during the 2002 outburst indicates that the WD is growing in mass.

\section{The Accreting White Dwarf in the Dwarf Nova U Geminorum}

The prototypical dwarf nova U Gem, along with a handful of other systems, reveal their underlying white dwarf photospheres during quiescence. This makes it possible to carry out detailed chemical abundance analyses like those reported by [20] for U Gem, VW Hyi and WZ Sge(see references therein), and [3] for the dwarf nova systems BW Scl, BC UMa and SW UMa. All of these object have suprasolar Al abundances in their photospheres and in some cases elevated Phosphorus. While the origin of these suprasolar abundances of odd-numbered nuclides remains a mystery, it is clear that to synthesize these heavy elements requires very high temperatures associated with explosive CNO burning [18]. Thus, it is likely that these photospheric overabundances are associated with the accreting white dwarf.

Recently, [6] reported the results of a study of the accreting white dwarf in U Gem as a function of time since a single dwarf nova outburst. This series of HST/COS spectra was intended to look for any variations of the metal abundances that could relate to accretion-diffusion equilibrium and other processes in the CV white dwarf photosphere. Our analysis confirms the high N/C ratio observed previously in the FUV spectra of U Gem in quiescence, a sign of possible CNO enrichment of the accreted material on the WD surface.

While the full analysis of the spectra remains in progress, the N/C abundance ratio is anomalously high as found by [19], the $\mathrm{N}$ abundance is $20 \mathrm{x}$ times solar, the $\mathrm{Al}$ abundance is 20 times solar and the likely detection of Argon with an abundance of 20 times solar. While it cannot be ruled out that the absorption lines might be forming in material veiling the WD rather than in the WD itself, if confirmed, this is the first detection of Argon in the photosphere of a CV white dwarf. The spectra also exhibit solar to sub-solar abundancesof carbon and sulfur, iron is only marginally supra-solar $\left(2[\mathrm{Fe}]_{\odot}\right)$, Silicon is sub-solar except for the Si III $(\sim 1112 \AA)$ and Si IV $(1400 \AA)$ that are solar. In Fig.3, we display the final COS spectrum of the time series obtained 56 days after the return to quiescence following a wide dwarf nova outburst of $\mathrm{U}$ Gem.

\section{Acknowledgments}

PG is pleased to thank William (Bill) P. Blair in the Henry A. Rowland Department of Physics \& Astronomy at the Johns Hopkins University, Baltimore, MD, USA, for his kind hospitality. This work was supported by the National Aeronautics and Space Administration (NASA) under grant number NNX17AF36G, HST grants GO-14641 and GO-14111, all to Villanova University

\section{References}

[1] G.C. Anupama, 2008, in: A. Evans, M. F. Bode, T. J. O’Brien \& M. J. Darnley eds., RS Ophiuchi (2006) \& the Recurrent Nova Phenomenon ASPCS 401, San Francisco, p.31 
U Gem
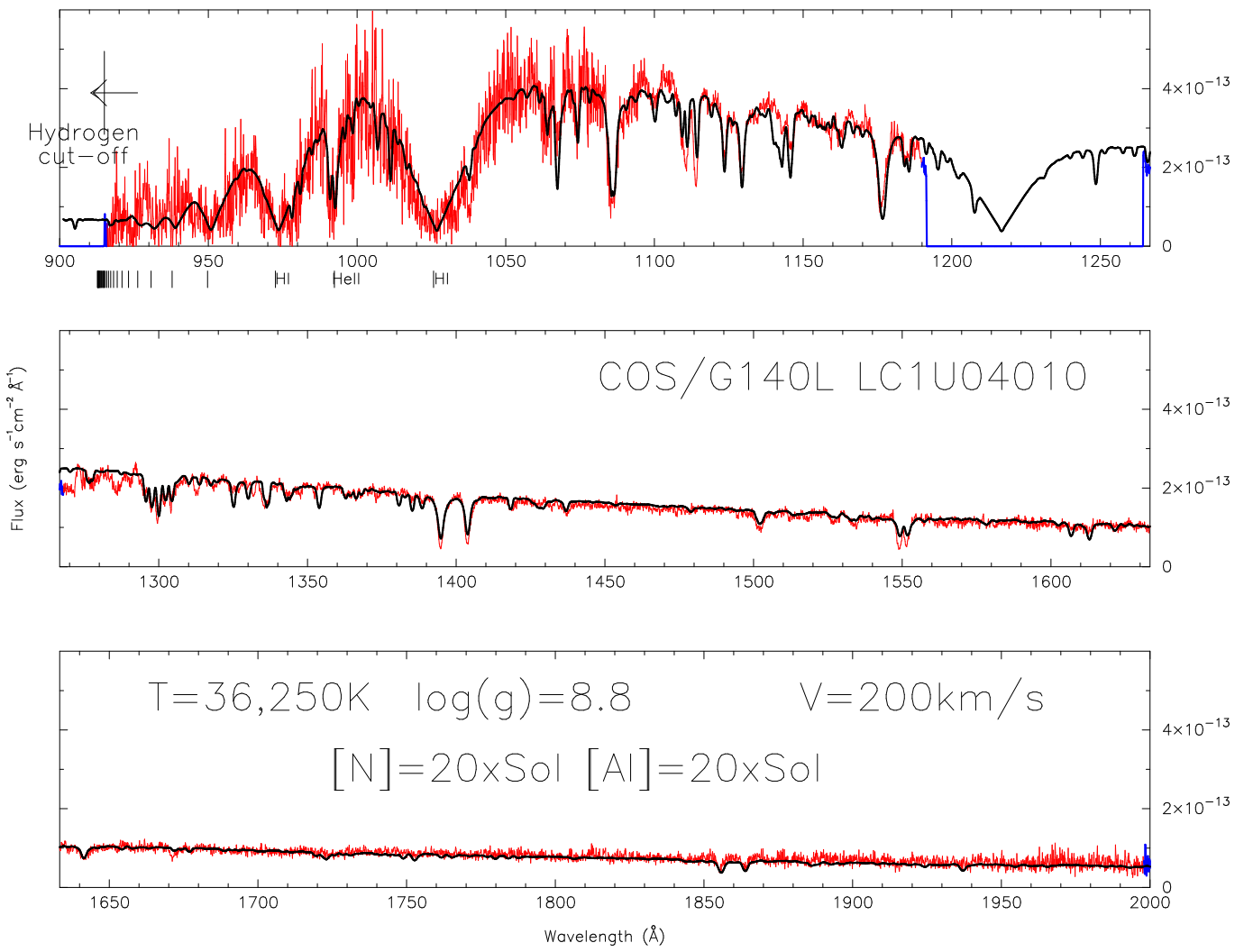

Figure 3: The COS spectrum of U Gem obtained 56 days after the peak of the outburst, modeled with a $36,250 \mathrm{~K}$ WD model. The spectrum was generated by co-adding the 4 sub-exposures obtained in one (HST) orbit.

[2] G.C. Anupama, 2013, in Binary Paths to Type Ia Supernovae Explosions, Proceedings of the International Astronomical Union, IAU Symposium, Volume 281, p. 193-194

[3] B.T. Gänsicke, et al. 2005, ApJ, 629, 451

[4] P. Godon, E.M. Sion, S. Starrfield, M. Livio, R.E. Williams, C. Woodward, P. Kuin, K. Page, 2014, ApJ, 784, 33

[5] P. Godon, M.M. Shara, E.M. Sion, D. Zurek, 2017, ApJ, 850, 146

[6] P. Godon, E.M. Sion, R.E. Williams, S. Starrfiled, 2018, ApJ,submitted

[7] J. Greiner, \& R. Di Stefano, 2002, ApJ, 578, L59

[8] T. Iijima, 2012, A\&A, 544, 26

[9] M. Kato, 2002, in B.T. Gänsicke, K. Beuermann \& K. Reinsch eds., The Physics of Cataclysmic Variables \& Related Objects, ASPCS 261, San Francisco, p.595

[10] C. Knigge, A.R. King, J. Patterson, 2000, A\&A, 364, L75

[11] C. Lederle, \& S. Kimeswenger, 2003, A\&A, 397, 951

[12] M. Orio, E. Tepedelenlioglu, S. Starrfield, et al. 2005, ApJ, 620, 938 
[13] A. Pagnotta, \& B.E. Schaefer, 2014, ApJ, 788, 164

[14] D.I. Sahman, V.S. Dhillon, 2013, in Binary Paths to Type Ia Supernovae Explosions, Proc. of the Intntl Astron. Union, IAU Symp., Vol.281, p. 193-194

[15] D.I. Sahman, et al. 2013, MNRAS, 433, 1588

[16] B.E. Schaefer, 2010, ApJS, 187, 275

[17] Sion, E.M., Godon, P., McClain, T. 2010, AAS 215:419.22, vol.42, p.280

[18] E.M. Sion, \& W.M. Sparks, 2014, ApJL, 796, L10

[19] E.M. Sion, et al. 1998, ApJ, 496, 449

[20] E.M. Sion, 1999, PASP, 111, 532

[21] J.L., Sokoloski, et al. 2013, ApJL, 770, L33

[22] S. Starrfield, W.M. Sparks, \& J.W. Truran, 1985, ApJ, 291, 136

[23] R.E. Wilson, \& R.K. Honeycutt, 2014, ApJ, 795,8

[24] P.A. Woudt, \& B. Warner, 2003, MNRAS, 343, 313

[25] O. Yaron, D. Prialnik, M.M. Shara, \& A. Kovetz, 2005, ApJ, 623, 398 